\title{
Phosphorene: An emerging 2D material
}

\author{
Kiho Cho, Jiong Yang, and Yuerui $\mathrm{Lu}^{\text {a) }}$ \\ Research School of Engineering, College of Engineering and Computer Science, The Australian National \\ University, Canberra, ACT 2601, Australia
}

(Received 20 December 2016; accepted 13 February 2017)

\begin{abstract}
Phosphorene has recently gained tremendous interest in the current decade, specifically, black phosphorus monolayer, a unique 2D material, investigation of which has led toward the creation of new scientific discoveries for future optoelectronic sensor devices. Beyond the success of graphene and other 2D layered materials research over the past decades, the increased interest toward this new emerging single-element structured material is because of its layer dependent $0.3-2.0 \mathrm{eV}$ band gap modulation range which is also the band gap modulation range of single- and few-layered graphene and transition metal dichalcogenides (TMDs). Besides that, phosphorene allows strong light-matter interactions at resonance because of its unique physical structure and outstanding electrical and optical properties. Therefore, current advancements are being done to enhance the performance of phosphorene thin films because of its applicability in different fields. This paper is aimed to highlight key properties, applications, and future perspects and challenges incurred regarding the use of $2 \mathrm{D}$ layered phosphorene.
\end{abstract}

\section{INTRODUCTION}

A well-known revolutionary 2-dimensional (2D) material graphene has been widely explored since its discovery to investigate its fundamental physics to find its applicability in practical applications. Among many remarkable properties, graphene's good transport properties, and high thermo-mechanical properties have proven its strong suitability in nanoelectronics and photonics. However, symmetrical electrical band structure of semi-metallic graphene at Dirac point, i.e., zero band gap energy limits its use for many optoelectronic applications. Therefore, many remarkable efforts have been done to increase its band gap $\left(E_{\mathrm{g}}\right) .{ }^{1-6}$ To address these issues, simultaneously, a lot of research has been made toward semiconducting materials such as transition metal dichalcogenides (TMDs) and insulating materials such as hexagonal boron nitride (h-BN), silicene, and phosphorene, at quite a rapid pace. Among 2D family of materials, phosphorene, a single atomic layer of black phosphorus (BP), has recently attracted significant attention due to its interesting strong angle dependent opto-electronic properties and anisotropic characteristics. In particular, by filling the large gap of unresolved properties such as energy gap, ${ }^{4,6-11}$ charge carrier mobility, ${ }^{12-16}$ current on/off ratio $^{16-18}$ and response time ${ }^{19,20}$ between graphene and

\footnotetext{
Contributing Editor: Venkatesan Renugopalakrishnan

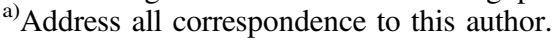

e-mail: yuerui.lu@anu.edu.au

DOI: $10.1557 / \mathrm{jmr} .2017 .71$
}

TMDs, this unique feature is coming to the forefront of next generation nano-sized electronic devices.

Above all, a widely tunable band gap $\left(E_{\mathrm{g}}\right)$ of phosphorene is one of the great advantages in 2D regimes, and this interesting electronic structure induces light-matter optical interaction with very broad spectral range from the visible to infrared region. Theoretical calculations ${ }^{21}$ and experimental data ${ }^{22-24}$ show that the layer-dependent direct $E_{\mathrm{g}}$ of black phosphorus (a few layer of phosphorene) compared to graphene and TMD semiconductors ranges broadly from 0.3 to $2.05 \mathrm{eV}$. In this sizable value, it can be further controlled using various methods, for instance, mechanical stress, ${ }^{25,26}$ electrical field, ${ }^{24}$ dimensional size control, ${ }^{27}$ edge oxidation, ${ }^{28,29}$ different stacking orders, ${ }^{30}$ hetero structure with other 2D materials, ${ }^{31,32}$ and substitutional doping. ${ }^{33,34}$ For layer dependency, the single layer has a noticeable $E_{\mathrm{g}} \sim 2.0 \mathrm{eV}$, but it gradually decreases to 0.3 $\mathrm{eV}$ as increasing film thickness to bulk size which is explained by the harmonized effect of interlayer coupling and quantum confinement effect. ${ }^{35}$ In addition, unlike the indirect to direct band gap transition in TMDs, the band structure of phosphorene remains direct at the $\Gamma$ point of Brillouin zone as increasing thickness up to few-layer. Only in bulk BP, conduction band minimum and valence band maximum are detected at the $Z$ point. This stable and thickness independent band topology is very beneficial for its potential photonics and optoelectronics in nano scale 2D regime.

The puckered lattice structure of phosphorene as shown in Figs. 1(a) and 1(b) leads to in-plane anisotropic physical properties including thermal conductivity, ${ }^{36}$ 


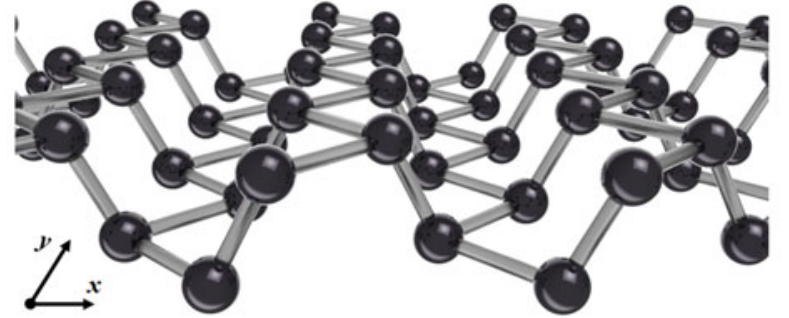

(a)

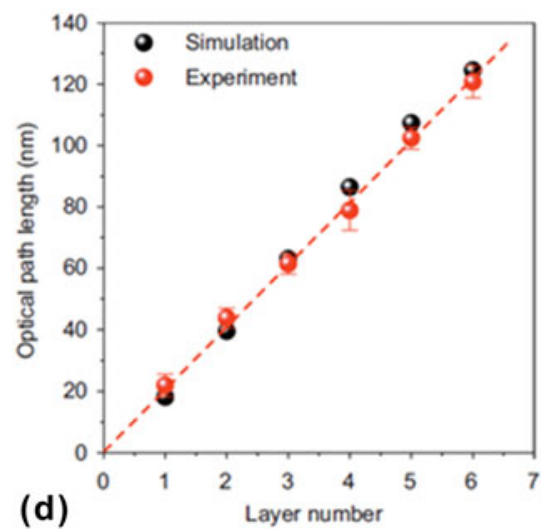

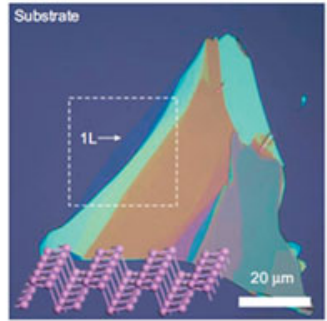

(b)

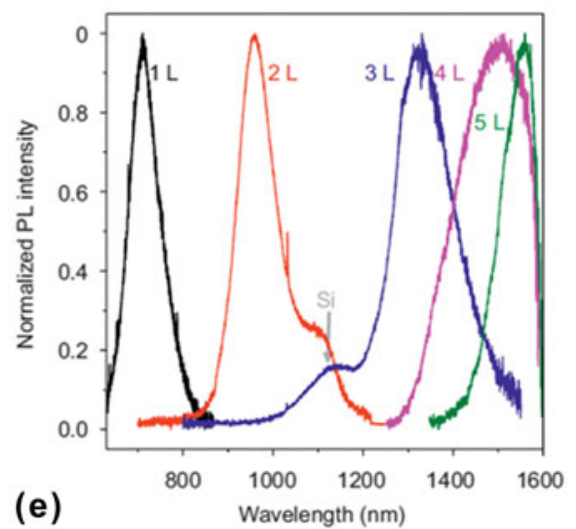

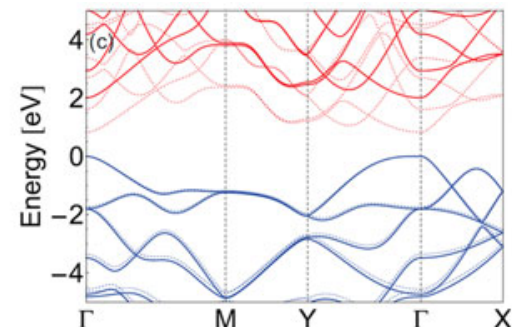

(c)

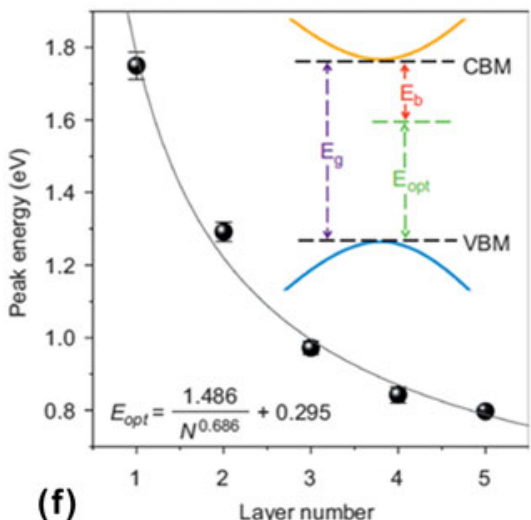

FIG. 1. Atomic structure and layer dependent characteristics of black phosphorus. (a) Schematic diagram of black phosphorus layer structure. (b) Microscope image of 1L phosphorene. (c) Calculated band structure by DFT (dashed lines) and GW-BSE (solid lines) for monolayer phosphorene. (d) OPL values from simulation (black dots) and experimental PSI measurements (red dots) for black phosphorus layers from 1L to 6L. (e) PL spectra of the $1 \mathrm{~L}-5 \mathrm{~L}$ phosphorene flakes. PL intensities are normalized, and the emission peak of the PL spectrum for monolayer is at $711 \mathrm{~nm}$, corresponding to peak energy of $1.75 \mathrm{eV}$. (f) Evolution of PL peak energy with layer number of phosphorene. The PL for the monolayer was measured at $-10{ }^{\circ} \mathrm{C}$, while others were measured at room temperature. Inset: schematic energy diagram showing the electronic band gap $\left(E_{\mathrm{g}}\right)$, optical gap $\left(E_{\mathrm{opt}}\right)$, and the exciton binding energy $\left(E_{\mathrm{b}}\right)$. Parts (b), (d), (e), and (f) are adapted with permission Ref. 54. Parts (c) is adapted with permission from Ref. 21.

Young's modulus ${ }^{18,25,37,38}$ carrier mobility and effective masses, ${ }^{38,39}$ and exciton dynamics, ${ }^{40-43}$ which are in marked contrast to those of graphene and TMDs. Highly anisotropic natures extended the prospect of phosphorene for a broad range of optical applications with a precedently unrevealed sizable band gap. Consequently, to optimize the designed phosphorene optical sensors with high-efficiency, it becomes critical to determine the right orientation on the substrates as well as to select the interlayer stacking orders. Notwithstanding the promising candidates of post graphene 2D materials as introduced, phosphorene is unstable and prone to oxidation promptly in ambient conditions in contrast to graphene, ${ }^{44}$ which degrades a lifecycle in most of electrical devices. Thus, new passivation methods were announced using $\mathrm{Al}_{2} \mathrm{O}_{3},{ }^{45-47}$ graphene, ${ }^{48} \mathrm{~h}-\mathrm{BN},{ }^{32}$ or hydrophobic polymer ${ }^{49-51}$ as a passivation layer, and more aggressively phosphorus oxide $\left(\mathrm{P}_{x} \mathrm{O}_{y}\right)$ layers similar to $\mathrm{SiO}_{2}$ on $\mathrm{Si}$ substrate could be used as a functional material in single- or few-layer at the pristine $\mathrm{BP}$ surface. $^{47}$

In this perspective, we present the recent advances in the unique optical characteristics of phosphorene, such as anisotropic luminescence, light-matter interaction, tunable
$E_{\mathrm{g}}$ and exciton emissions, and also discuss challenges for its practical applications to current nano-technology. Finally, the state of the art of opto-electrical sensing devices based on BP is introduced.

\section{ELECTRICAL STRUCTURE AND LAYER DEPENDENT OPTICAL PROPERTIES}

Bulk black phosphorus (BP) consists of infinite number of phosphorene monolayers similar to graphene and other 2D materials, and the 2D flat layers are weakly coupled by the van der Waals force which allows to exfoliate BP into phosphorene nano-sheets. ${ }^{52}$ However, unlike in other 2D materials, phosphorene does not form entirely flat sheets like but it constructs a puckered surface structure due to the $s p^{3}$ hybridization. Based on pre-revealed 2D materials atomic crystalline structure, phosphorene's ridged surface is normally defined as $x$ axis-zigzag direction and $y$ axis-armchair direction.

From the perspective of semiconducting applications, since the electronic band gap structure is a fundamental factor, it is theoretically calculated by using first-principles density functional theory (DFT) and GW-Bethe-Salpeter equation (BSE) [Fig. 1(c) $]^{21}$ and also experimentally 
measured by using photoluminescence excitation spectroscopy ranging from $0.3 \mathrm{eV}$ in bulk to $\sim 2.0 \mathrm{eV}$ in monolayer. This sizable value is obviously thickness dependent, and induced exciton binding energy and optical absorption are also controllable with different thickness in contrast to other 2D layered materials.

Few-layer phosphorene has only several atoms in thickness, but the PL intensities from $2 \mathrm{~L}$ to $5 \mathrm{~L}$ layered phosphorene are all stronger than that from the thick $\mathrm{Si}$ substrate. This is because of the direct band gap structure in few-layer phosphorene and the indirect band gap in $\mathrm{Si}$. As compared to the indirect band gap behaviors in TMDs, ${ }^{8,53}$ this intrinsic direct band gap nature in fewlayer phosphorene serves them great potential advantages for various optoelectronic applications.

Layer thickness of nano-sheets can be calculated using Kelvin probe force microscope (KPFM) but it has a risk to damage the delicate sample surfaces by the sharp scanning tip during the measurement. Recently, optical path length (OPL) of the light reflected from the 2D layer is introduced as a fast, non-invasive, and highly accurate technique to measure the thickness and the atomic layer number of BP. ${ }^{54}$ The OPL is calculated from the relation, $\mathrm{OPL}_{\mathrm{BP}}=-\frac{\lambda}{2 \pi}\left(\varnothing_{\mathrm{BP}}-\varnothing_{\mathrm{SiO}_{2}}\right)$, where $\lambda$ is the wave length of the light source, and $\varnothing_{\mathrm{BP}}$ and $\varnothing_{\mathrm{SiO}_{2}}$ are the phase shift of the light reflected from the $\mathrm{BP}$ and $\mathrm{SiO}_{2} / \mathrm{Si}$ substrate, respectively. In experiment, phase-shifting interferometry (PSI) using very low-density light from a light-emitting diode (LED) is used to measure the OPL. OPL values obtained from simulations and experiments for BP from 1L to 6L are linearly fit well as shown in Fig. 1(d).

With the evolution of the electronic band structure on low-dimensional materials, the induced exciton, a bound quasiparticle state between electron and hole through a Coulomb force, plays a key role to understand the relation between variant effects of the spacial dimension, shape, crystalline structure, and material composition on the nano-optoelectronic properties. Figure 1(e) shows the normalized PL spectra of the $1 \mathrm{~L}-5 \mathrm{~L}$ phosphorene flakes. The emission peak of the PL spectrum for monolayer phosphorene is at $711 \mathrm{~nm}$, corresponding to the peak energy of $1.75 \mathrm{eV}$. The peak energy of PL emission, which is termed as optical gap $\left(E_{\text {opt }}\right)$, is the difference between the electronic band gap $\left(E_{\mathrm{g}}\right)$ and the exciton binding energy $\left(E_{\mathrm{b}}\right)$ [Fig. 1(f) inset]. Strong quantum confinement effect at free-standing monolayer phosphorene induces a large exciton binding energy of $\sim 0.8 \mathrm{eV},{ }^{21,55}$ whereas this value for monolayer phosphorene on a $\mathrm{SiO}_{2} / \mathrm{Si}$ substrate is measured to be only $\sim 0.3 \mathrm{eV}$ on account of the increased screening effect from the substrate. ${ }^{55} \mathrm{~A}$ high exciton binding energy stabilizes excitons and trions (charged excitons) against thermal fluctuations which conventionally degrade the transport efficiency in opto-electronic devices.
As depicted in Fig. 1(f), the optical gaps in phosphorene layers increase rapidly with decreasing layer number because of the strong quantum confinement effect and the van der Waals force between the phosphorene layers. ${ }^{54}$ A nonlinear curve by fitting the measured optical gaps is obtained as $E_{\mathrm{opt}}=\frac{1.486}{N^{0.686}}+0.295$, where $E_{\mathrm{opt}}$ is the optical gap in unit of $\mathrm{eV}$ and $N$ is the layer number, and this layer-dependent optical gap function agree well with the theoretical models. ${ }^{21}$ For bulk phosphorene sample with infinite $N$ value, its optical gap converges the limit value of $\sim 0.295 \mathrm{eV}$, which perfectly matches well with the measured energy gap $(\sim 0.3 \mathrm{eV})$ of bulk phosphorus. ${ }^{56,57}$

\section{ANISOTROPIC OPTICAL PROPERTIES}

The atomic crystal structure of phosphorene possesses a puckered orthorhombic lattice with the $D_{2 \mathrm{~h}}$ group point symmetry. ${ }^{58}$ The lattice parameters along the two perpendicular directions are respectively $\left|a_{1}\right|=4.37 \AA$, $\left|a_{2}\right|=3.31 \AA^{58}{ }^{58}$ Due to its notable nonplanar, ridged structural shape, phosphorene shows anisotropic natures of electronic, optoelectronic, phononic, and thermoelectronic properties. These properties are principally affected by the carrier mobility $(\mu)$, which is inversely proportional to the effective masses $\left(m^{*}\right)$ as $=q \tau / m^{*}$, where $\tau$ is the scattering time and $q$ is the charge. The anisotropic effective mass values of carriers along zigzag $(x)$ and armchair $(y)$ directions at phosphorene are calculated ${ }^{39}: m^{*}=\hbar^{2}\left(\frac{\partial^{2} E(k)}{\partial k^{2}}\right)^{-1}$, where $k$ is the reciprocal lattice vector and $E$ is the energy, and $m_{\mathrm{c} \text {, zig }}^{*}$ is up to $\sim 10$ times heavier than $m_{\mathrm{c} \text {, arm }}^{*}$ indicating that the armchair direction is much more efficient for carrier transport.

The polarization angle dependency of phonon dynamics was simply measured using Raman spectroscopy. As shown in Fig. 2(a), Raman scattering intensities exhibit $\mathrm{A}_{\mathrm{g}}^{1}, \mathrm{~B}_{2 \mathrm{~g}}$, and $\mathrm{A}_{\mathrm{g}}^{2}$ phonon modes along the $z, y$, and $x$ directions in phosphorene flakes respectively. Intensity of $\mathrm{B}_{2 \mathrm{~g}}$ (armchair) and $\mathrm{A}_{\mathrm{g}}^{2}$ (zigzag) modes are significantly dependent on the polarization angle by showing an angle period of $180^{\circ}$ [Fig. 2(b)]. Each two modes generate maximum value when the laser polarization is parallel $\left(0^{\circ}\right)$ with its crystalline direction, while the intensity reaches minimum when it is crossing right angle $\left(90^{\circ}\right)$. However, since $A_{g}^{1}$ mode oscillates along $z$-direction, it is not sensitive to the angle of laser polarization. ${ }^{40}$ Furthermore, the labdesigned polarized optical microscope revealed optical anisotropy of few-layer $\mathrm{BP}$ in the visible regime. ${ }^{59}$ Optical contrast in the RGB channels of BP exhibits periodic variation with the rotation angles from 0 to $180^{\circ}$ [Figs. 2(c) and 2(d)]. These simple and accurate methods are optimized to determine the atomic crystalline orientation of phosphorene layers, and it will 

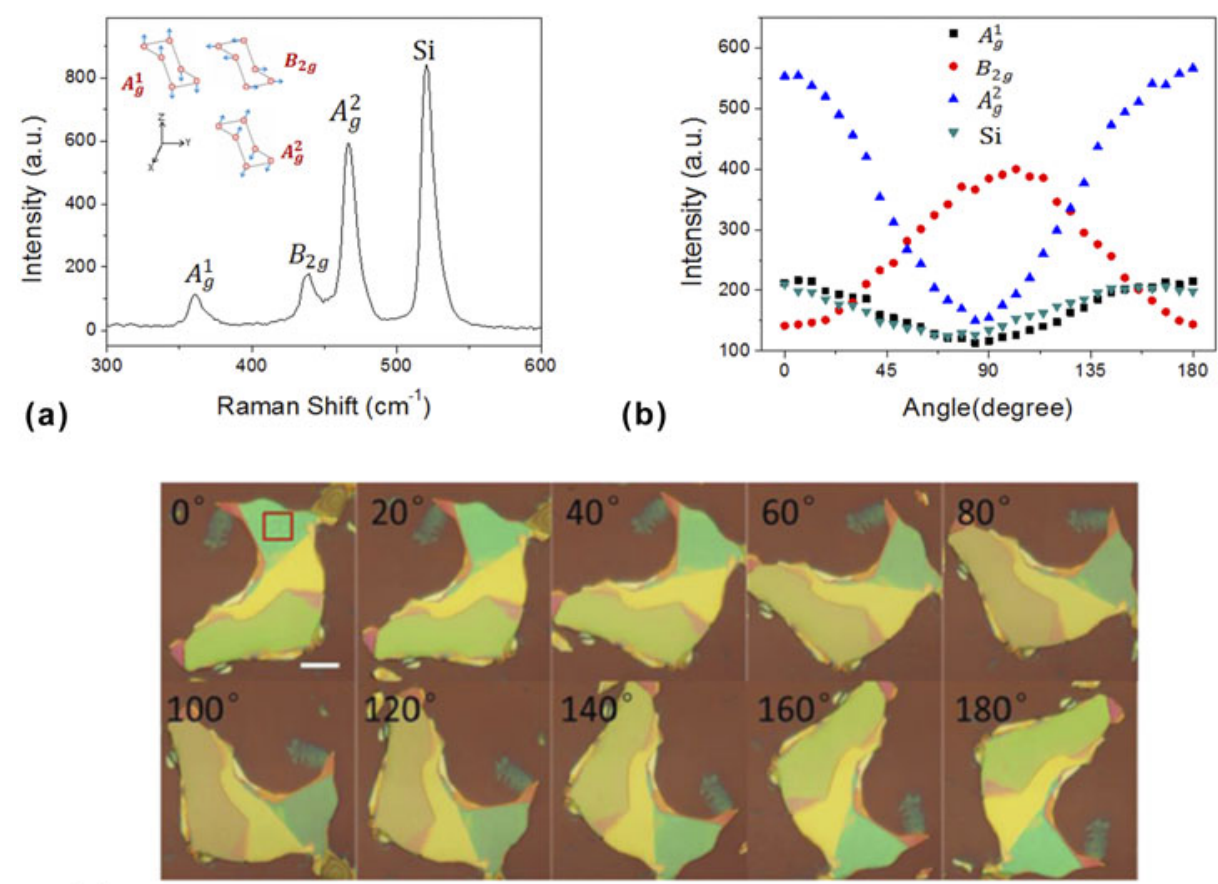

(c)
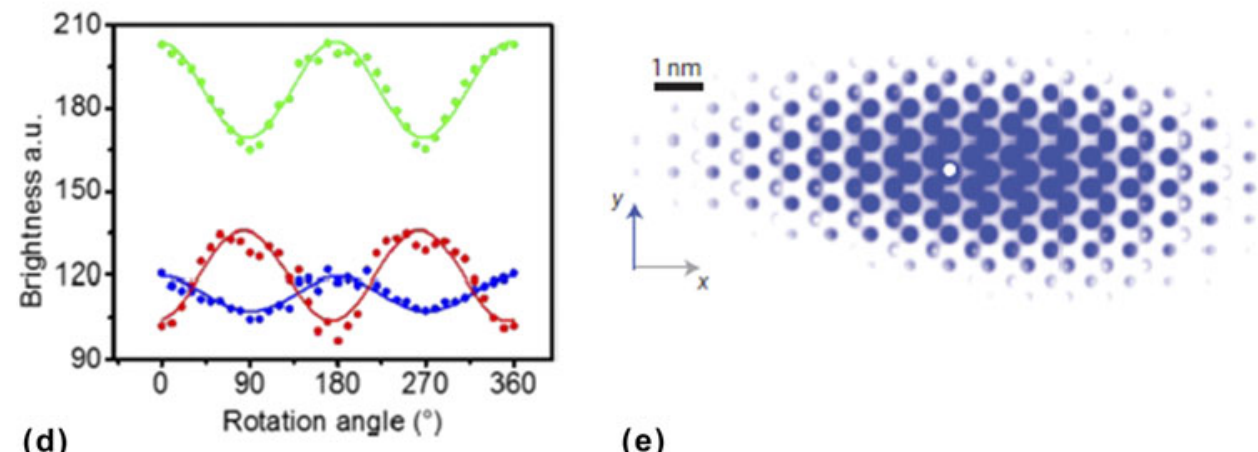

(d)

Rotation angle $\left({ }^{\circ}\right)$

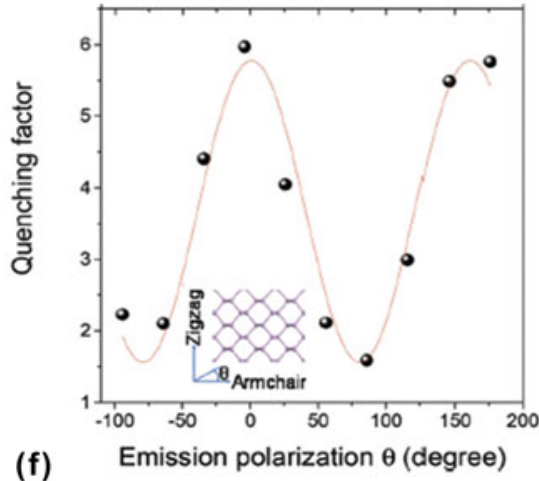

(e)

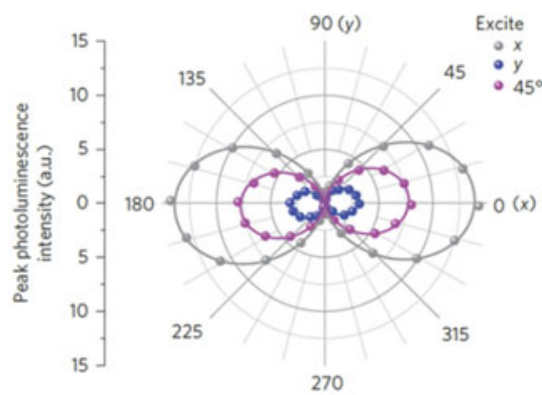

(g)

FIG. 2. Anisotropic optical properties of black phosphorus. (a) Raman spectrum of $2 \mathrm{~L}$ phosphorene. Inset: the vibration directions of $\mathrm{A}_{\mathrm{g}}^{1}$, $\mathrm{B}_{2 \mathrm{~g}}$, and $\mathrm{A}_{\mathrm{g}}^{2}$ Raman modes. (b) Polarization dependence of $\mathrm{A}_{\mathrm{g}}^{1}, \mathrm{~B}_{2 \mathrm{~g}}$, and $\mathrm{A}_{\mathrm{g}}^{2}$ modes in a $15 \mathrm{~L}$ phosphorene and silicon substrate. The intensities of $\mathrm{B}_{2 \mathrm{~g}}$ and $\mathrm{A}_{\mathrm{g}}^{2}$ modes both show the apparent angle dependent curves. (c) Optical images of a thick black phosphorus sample shows a color change as a function of rotation angle, and (d) RGB optical intensities of the reflected light in the region marked with red square in (c) exhibit sinusoidal behaviors. (e) Top view of the square of the electron wavefunction of the bright ground-state exciton in monolayer phosphorene. Since the carriers are more mobile along the $x$ direction with low effective mass and the Coulomb interaction is isotropic, the exciton is anisotropic forming striped patterns. (f) The measured PL quenching factor from the $2 \mathrm{~L}$ phosphorene. The red line is the fitted sinusoidal function of emission polarization angle. (g) Photoluminescence peak intensity as a function of polarization detection angle for excitation laser polarized along $x$ (gray), $45^{\circ}$ (magenta) and $y$ (blue) directions. Parts (a) and (b) are adapted with permission Ref. 40. Parts (c) and (d) are adapted with permission Ref. 59. Parts (e) and (g) are adapted with permission Ref. 41. Part (f) is adapted with permission with Ref. 23. 
touch up the nano-measurement technologies for other anisotropic 2D layered structures.

In particular, excitons in phosphorene are predicted to be confined in a quasi-one-dimensional (1D) space due to its anisotropic nature, in contrast to other 2D materials such as graphene and TMDs, so it provides an ideal platform for investigating remarkable exciton dynamics in a reduced regime. Especially, excitons at phosphorene are characterized that their spatial distribution of wave functions is anisotropic by intensely extending along the armchair direction. ${ }^{41}$ Figure 2(e) displays the simulated wave function of the bright ground-state exciton. This result is associated with the consequence of the directional mass dependence, and the isotropic Coulomb interaction binds the carriers most strongly in the zigzag direction. Experimentally, the excitons are measured by capturing the linearly polarized excitons using angle-resolved PL spectroscopy. For example, charge transfer dynamics has been determined in a phosphorenegold hybrid system where the bilayered phosphorene flake is transferred on a gold pad, which is patterned on $\mathrm{SiO}_{2} / \mathrm{Si}$ substrate. ${ }^{23}$ The maximum PL intensities at $945 \mathrm{~nm}$ from phosphorene on $\mathrm{Au}$ and $\mathrm{SiO}_{2} / \mathrm{Si}$ substrate show a big different value and large in-plane anisotropy along the

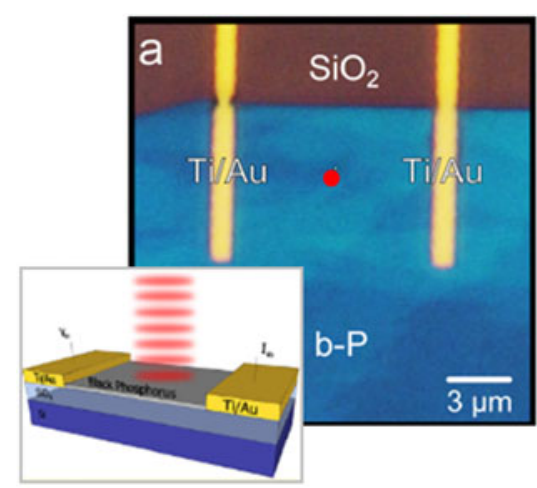

(a)

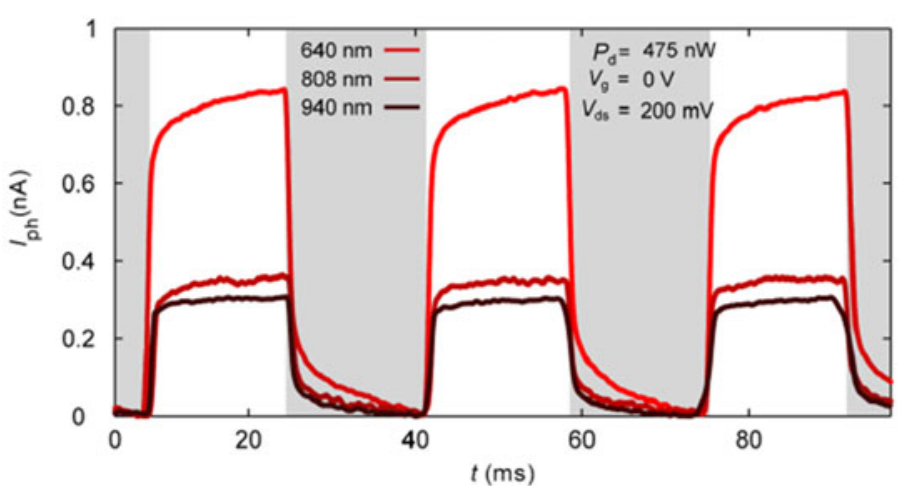

(b)
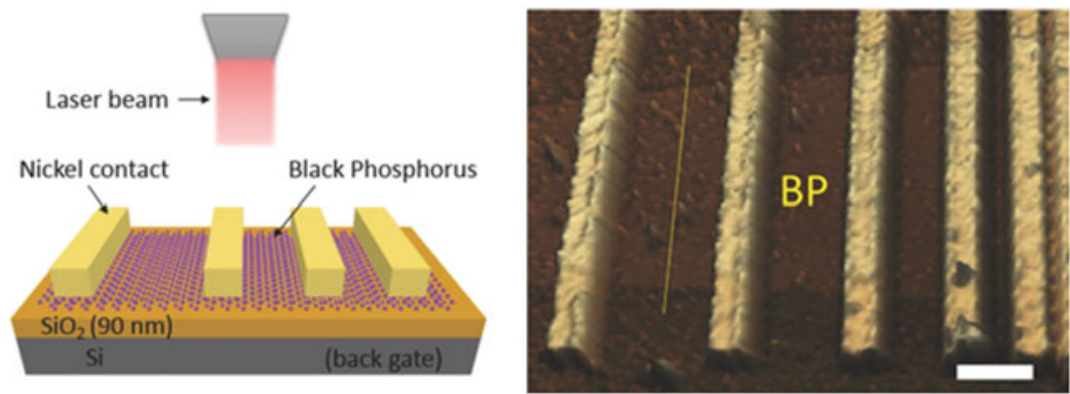

(e) (d)

(c)
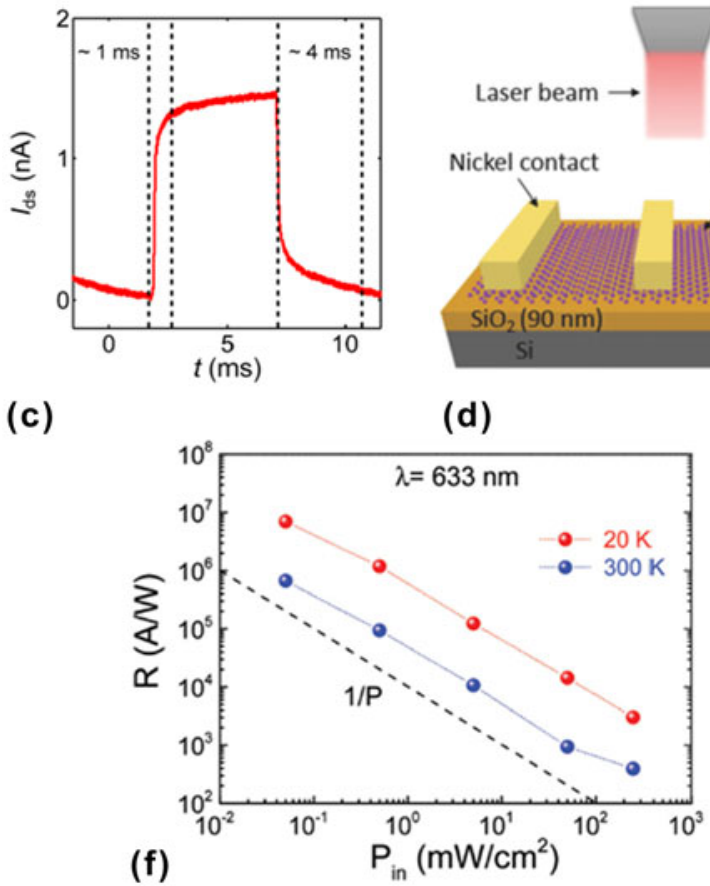

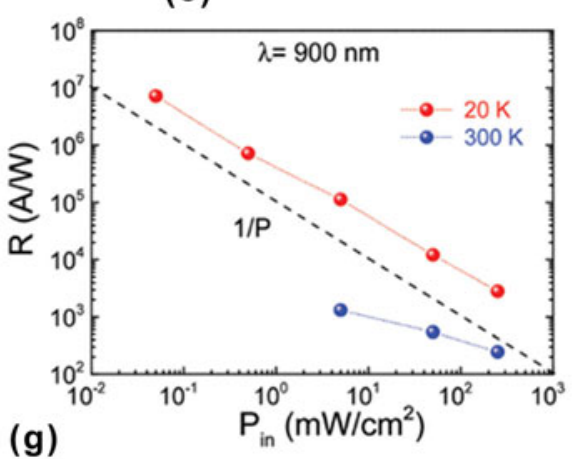

(g)

FIG. 3. Black phosphorus (BP) based photo-detecting sensors. (a) Configuration of the fast photoresponse sensing device based on BP-FET. (b) Measured photocurrent responses from the BP-FET for different wavelengths $(\lambda) 640,808$, and $940 \mathrm{~nm}$. Peak value decreases with increasing wave length, and (c) photocurrent intensity in one period of light excitation $\left[\lambda=640 \mathrm{~nm}\right.$, incident power $\left.\left(P_{\mathrm{d}}\right)=6.49 \mu \mathrm{W}\right]$ exhibits very fast rise and fall response time (1 ms and $4 \mathrm{~ms}$ ). (d) BP based photodetector with high responsivity $(R)$ and (e) its 3D topography. (f and g) Responsivity of the detector at different incident laser power. The wave length of the laser is 633 and $900 \mathrm{~nm}$ in part (f) and (g), respectively. A high photoresponsivity $R=6.7 \times 10^{5} \mathrm{~A} / \mathrm{W}$ for $633 \mathrm{~nm}$, and $7.0 \times 10^{6} \mathrm{~A} / \mathrm{W}$ for $900 \mathrm{~nm}$ both at $20 \mathrm{~K}$ condition. Parts (a), (b), and (c) are adapted with permission Ref. 20. Parts (d), (e), (f), and (g) are adapted with permission Ref. 60. 
emission polarization angle. A quenching factor defined by $\frac{\mathrm{PL} \text { intensity on } \mathrm{SiO}_{2} / \mathrm{Si}}{\mathrm{PL} \text { intensity on } \mathrm{Au}}$ is used to describe the exciton dynamics, and the plotted data presents a well fitted curve by a sinusoidal function with a period of $\sim 180^{\circ}$ as shown in Fig. 2(f). Simultaneously, anisotropic nature of trions have also been observed with an extraordinarily large binding energy of $\sim 162 \mathrm{meV}^{42}$ which is approximately 1 order of magnitude higher than those in 2D TMDs. ${ }^{40,56}$ Furthermore, this intriguing PL observations are consistent in monolayer phosphorene, ${ }^{41}$ in which the PL intensity along the $y$ direction is steadily less than $3 \%$ of that along the $x$ direction [Fig. 2(g)] regardless of the excitation laser polarization.

Basically these anisotropic properties are originated from the unique atomic structure of phosphorene. Additionally, apart from those distinct electro-optical properties, phosphorene also exhibits strong directional dependent physical properties in thermal conductivity and mechanical strain behaviors. By understanding the anisotropic nature of this emerging material, we can discover more interesting physical phenomena in $2 \mathrm{D}$ layered heterostructure and its potential applications.

\section{HIGH-PERFORMANCE PHOTO DETECTORS BASED ON BLACK PHOSPHOROUS}

Intensive research efforts on the $\mathrm{BP}$ have led to high-performance photo sensing devices. As described on this Review, black phosphorus (BP) can satisfy both criterions on the electric- and optical-requirements as a next generation material with far-reaching potentials. Basically, photodetection ability relies on the transformation of absorbed photons into an electrical signal. Highly advanced photodetector based on FET using fewlayered BP (Fig. 3(a)) presents a remarkably fast time response $^{20}$ : rise $\left(\tau_{\text {rise }}\right)$ and fall $\left(\tau_{\text {fall }}\right)$ time to be $1 \mathrm{~ms}$ and $4 \mathrm{~ms}$, respectively as shown in Fig. 3(c). According to the wide range of the tunable band gap on $\mathrm{BP}$, photodetector operates across the visible and up to the near-infrared (NIR) regions (940 nm) [Fig. 3(b)]. However, as summarized in Table I, TMDs and semiconducting layered materials exhibit relatively slow photoresponse because of the large band gap; they are limited near the visible range of the spectrum.

Recently, BP photodetector based on backgate FET high photoresponsivity ${ }^{60}$ in a broad range from visible $(400 \mathrm{~nm})$ to NIR $(900 \mathrm{~nm})$ has been introduced as shown in Figs. 3(d) and 3(e). They drastically increased the level of photoresponsivity to be $7 \times 10^{6} \mathrm{~A} / \mathrm{W}$ at $20 \mathrm{~K}$ and $4.3 \times 10^{6} \mathrm{~A} / \mathrm{W}$ at $300 \mathrm{~K}$ for the $100 \mathrm{~nm}$ channel length in a broadband spectrum [Figs. 3(f) and 3(g)]. This device shows also high response time (rise) with $\tau_{\text {rise }}=5 \mathrm{~ms}$. A simplified relationship of responsivity against channel length agrees well with the experimental measurement, since the photoresponsivity $(R)$ can be expressed as $R \propto \frac{\tau_{\text {lifetime }}}{\tau_{\text {transit }}}$, where $\tau_{\text {lifetime }}$ is the lifetime of the photoinduced carrier, $\tau_{\text {transit }}$ is the carrier transfer time. Here, the transient time is provided by $\tau_{\text {transit }}=\frac{L^{2}}{\mu V_{\mathrm{ds}}}$, where $L$ is the channel length, $\mu$ is the carrier mobility, and $V_{\mathrm{ds}}$ is the drain-source voltage.

\section{v. CHALLENGES AND FUTURE RESEARCH DIRECTIONS}

Despite of the fact that phosphorene can be a new material in the 'post-graphene age' it is relatively vulnerable against environmental attack due to its tendency to oxidize in ambient conditions. ${ }^{44,61}$ To lay groundwork for the future prosperity of BP based sensors and its application techniques, some novel passivation techniques must be introduced. Among the many capping material candidates, graphene, h-BN, $\mathrm{AlO}_{x}$, and $\mathrm{Al}_{2} \mathrm{O}_{3}, \mathrm{P}_{x} \mathrm{O}_{y}$, and polymeric material such as PMMA [Figs. 4(d) and 4(e) $]^{50}$ are used to protect from mechanical and chemical degradation so far. A highly controllable $\mathrm{Al}_{2} \mathrm{O}_{3}$ coating method is developed, allowing us to fabricate high quality and airstable BP layers with a designed thickness from few-down to mono-layer. ${ }^{47}$ Shown in Fig. 4(a) is the schematic of passivation procedure. $\mathrm{O}_{2}$ plasma etching exfoliates the BP layers mechanically and also oxidizes top layers by protecting remained layers. The dynamic equilibrium between oxidation growth at the bottom of $\mathrm{P}_{x} \mathrm{O}_{y}$ and physical removal of the top enables to generate constant etching rate, then any designed layer thickness can be

TABLE I. Comparison of figures-of-merit for photodetectors based on 2D materials. Adapted from Ref. 20 with permission.

\begin{tabular}{|c|c|c|c|c|c|}
\hline Material & Measurement conditions & Responsivity (mA/W) & Response time (ms) & Spectral range & Reference \\
\hline$>1 \mathrm{~L} \mathrm{~b}-\mathrm{P}$ & $V_{\mathrm{ds}}=02 \mathrm{~V}, V_{\mathrm{g}}=0 \mathrm{~V}, \lambda=640 \mathrm{~nm}, P_{\mathrm{d}}=10 \mathrm{nW}$ & 4.8 & 1 & Visible-NIR & This work \\
\hline $1 \mathrm{~L} \mathrm{MoS}_{2}$ & $V_{\mathrm{ds}}=8 \mathrm{~V}, V_{\mathrm{g}}=-70 \mathrm{~V}, \lambda=561 \mathrm{~nm}, P=150 \mathrm{pW}$ & $880 \times 10^{3}$ & 600 & Visible & 29 \\
\hline $1 \mathrm{~L} \mathrm{MoS} 2$ & $V_{\mathrm{ds}}=1 \mathrm{~V}, V_{\mathrm{g}}=50 \mathrm{~V}, \lambda=532 \mathrm{~nm}, P=80 \mu \mathrm{W}$ & 8 & 50 & Visible & 8 \\
\hline$>1 \mathrm{~L} \mathrm{MoS}_{2}$ & $V_{\mathrm{ds}}=1 \mathrm{~V}, V_{\mathrm{g}}=-2 \mathrm{~V}, \lambda=633 \mathrm{~nm}, P=50 \mathrm{~mW} / \mathrm{cm}^{2}$ & 110 & $>10^{3}$ & Visible-NIR & 34 \\
\hline$>1 \mathrm{~L} \mathrm{WS}_{2}$ & $V_{\mathrm{ds}}=30 \mathrm{~V}, V_{\mathrm{g}}=\mathrm{N} / \mathrm{A}, \lambda=458 \mathrm{~nm}, P=2 \mathrm{~mW}$ & $21.4 \times 10^{-3}$ & 5.3 & Visible & 35 \\
\hline$>1 \mathrm{~L} \mathrm{In}{ }_{2} \mathrm{Se}_{3}$ & $V_{\mathrm{ds}}=5 \mathrm{~V}, V_{\mathrm{g}}=0 \mathrm{~V}, \lambda=300 \mathrm{~nm}, P=2.08 \mathrm{~W} / \mathrm{m}^{2}$ & $395 \times 10^{3}$ & 18 & UV-NIR & 33 \\
\hline$>1 \mathrm{~L} \mathrm{GaTe}$ & $V_{\mathrm{ds}}=5 \mathrm{~V}, V_{\mathrm{g}}=0 \mathrm{~V}, \lambda=532 \mathrm{~nm}, P=3 \times 10^{-5} \mathrm{~mW} / \mathrm{cm}^{2}$ & $10^{7}$ & 6 & Visible & 43 \\
\hline$>1 \mathrm{~L} \mathrm{GaSe}$ & $V_{\mathrm{ds}}=5 \mathrm{~V}, V_{\mathrm{g}}=0 \mathrm{~V}, \lambda=254 \mathrm{~nm}, P=1 \mathrm{~mW} / \mathrm{cm}^{2}$ & $2.8 \times 10^{3}$ & 300 & UV-visible & 36 \\
\hline$>1 \mathrm{~L} \mathrm{GaS}$ & $V_{\mathrm{ds}}=2 \mathrm{~V}, V_{\mathrm{g}}=0 \mathrm{~V}, \lambda=254 \mathrm{~nm}, P=0.256 \mathrm{~mW} / \mathrm{cm}^{2}$ & $4.2 \times 10^{3}$ & 30 & UV-visible & 32 \\
\hline
\end{tabular}



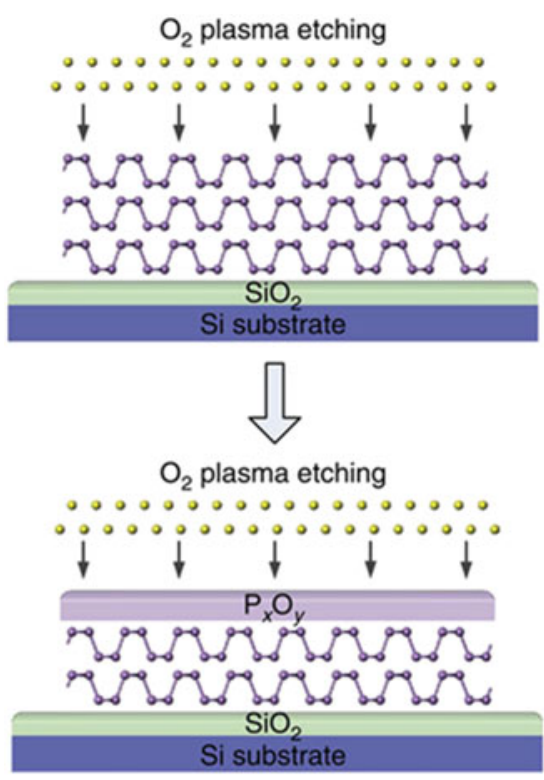

(a)

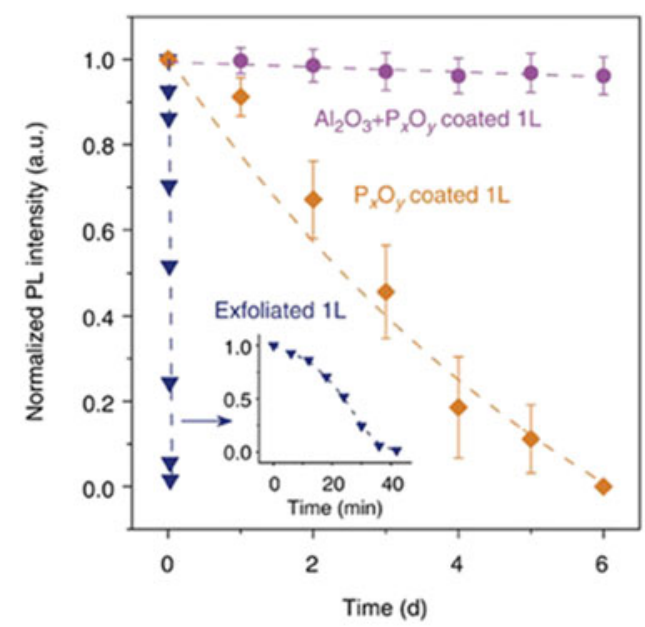

(b)

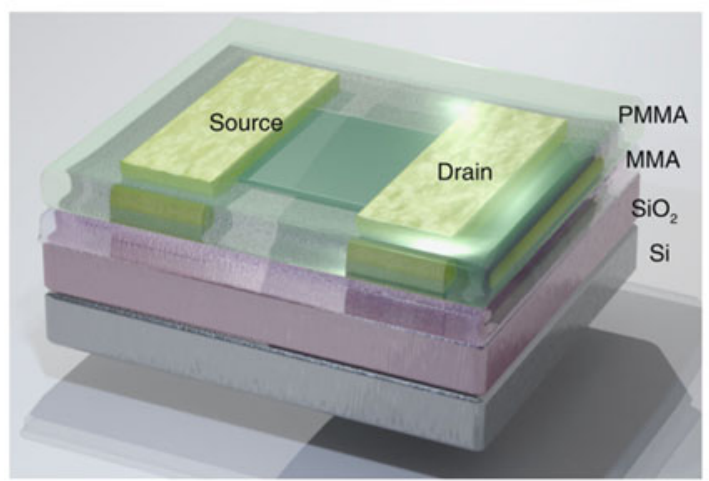

(d)
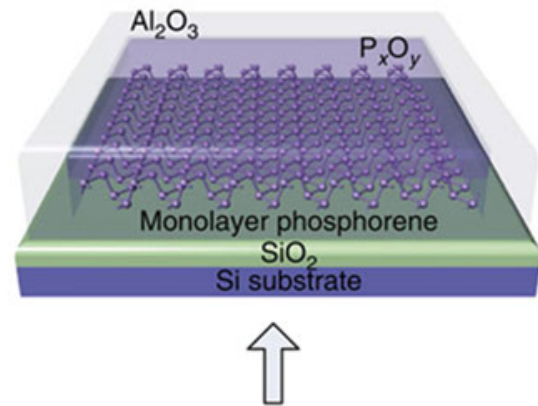

$\mathrm{ALD} \mathrm{Al} \mathrm{O}_{3}$ coating
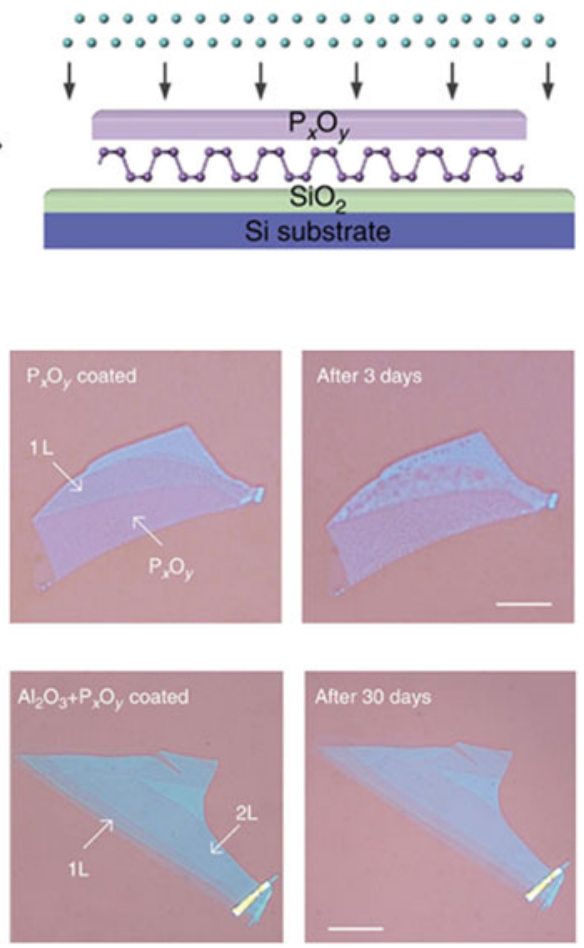

(c)

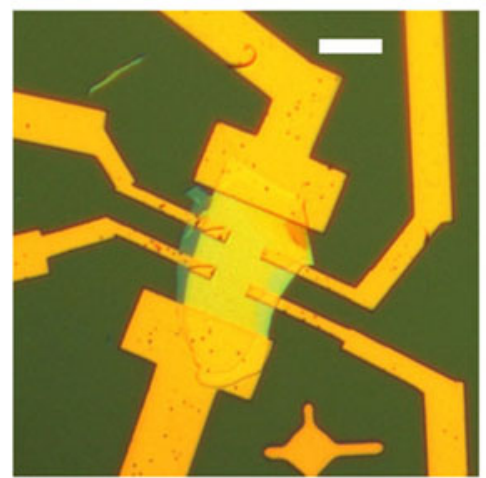

(e)

FIG. 4. Stability of black phosphorus. (a) Fabrication process of air-stable mono- and few-layer phosphorene sample. Accurate $\mathrm{O}_{2}$ plasma etching assists to create $\mathrm{P}_{x} \mathrm{O}_{y}$ protection layer and also to control the thickness of pristine phosphorene layer. $\mathrm{Al}_{2} \mathrm{O}_{3}$ capping layer deposited by ALD further increases life time of phosphorene sample. (b) Time dependence of the PL intensity and (c) microscopic surface images prove the stabilizing capability of the combined $\mathrm{Al}_{2} \mathrm{O}_{3}$ and $\mathrm{P}_{x} \mathrm{O}_{y}$ protective layers. (d) Schematic of a BP-FET with encapsulating layer of MMA and PMMS, and optical image of an encapsulated BP-FET (e). Parts (a), (b), and (c) are adapted with permission Ref. 47. Parts (d) and (e) are adapted with permission Ref. 50. 
fabricated. Finally the stabilized sample is capped by $\mathrm{Al}_{2} \mathrm{O}_{3}$ using atomic layer deposition (ALD) to increase the durability more. The sample with dual layers of $\mathrm{P}_{x} \mathrm{O}_{y}$ and $\mathrm{Al}_{2} \mathrm{O}_{3}$ have much longer lifetime with steady PL intensity in high level, as shown in Figs. 4(b) and 4(c), without any surface damages even after 2 months in air.

Various coupling effects of BP with other 2D layered materials have been remained as unveiled area. Their heterostructures and integrated hybrid systems are still an unexplored field. Furthermore, if uniform thickness controllability in large scale production of BP films is realized, it might trigger more intensive researches on BP materials and will rapidly open large commercial application markets such as opto-electronics, bio-sensing, optical communication, and wearable photonic devices.

\section{ACKNOWLEDGMENT}

We acknowledge financial support from the Australian Research Council (grant number DE140100805) and the ANU Major Equipment Committee.

\section{REFERENCES}

1. S.Y. Lee, D.L. Duong, Q.A. Vu, Y. Jin, P. Kim, and Y.H. Lee: Chemically modulated band gap in bilayer graphene memory transistors with high on/off ratio. ACS Nano 9(9), 9034 (2015).

2. S.J. Zhang, S.S. Lin, X.Q. Li, X.Y. Liu, H.A. Wu, W.L. Xu, P. Wang, Z.Q. Wu, H.K. Zhong, and Z.J. Xu: Opening the band gap of graphene through silicon doping for the improved performance of graphene/GaAs heterojunction solar cells. Nanoscale 8(1), 226 (2016)

3. M. Papagno, S. Rusponi, P.M. Sheverdyaeva, S. Vlaic, M. Etzkorn, D. Pacilé, P. Moras, C. Carbone, and H. Brune: Large band gap opening between graphene dirac cones induced by $\mathrm{Na}$ adsorption onto an Ir superlattice. ACS Nano 6(1), 199 (2012).

4. M. Dvorak, W. Oswald, and Z. Wu: Bandgap opening by patterning graphene. Sci. Rep. 3, 2289 (2013).

5. S.Y. Zhou, G.H. Gweon, A.V. Fedorov, P.N. First, W.A. de Heer, D.H. Lee, F. Guinea, A.H. Castro Neto, and A. Lanzara: Substrateinduced bandgap opening in epitaxial graphene. Nat. Mater. 6(10), 770 (2007)

6. M.Y. Han, B. Ozyilmaz, Y. Zhang, and P. Kim: Energy band-gap engineering of graphene nanoribbons. Phys. Rev. Lett. 98(20), 206805 (2007)

7. A.K. Geim and K.S. Novoselov: The rise of graphene. Nat. Mater. 6(3), 183 (2007).

8. A. Ramasubramaniam, D. Naveh, and E. Towe: Tunable band gaps in bilayer transition-metal dichalcogenides. Phys. Rev. B: Condens. Matter Mater. Phys. 84(20), 205325 (2011).

9. A. Chernikov, T.C. Berkelbach, H.M. Hill, A. Rigosi, Y. Li, O.B. Aslan, D.R. Reichman, M.S. Hybertsen, and T.F. Heinz: Exciton binding energy and nonhydrogenic Rydberg series in monolayer $\mathrm{WS}_{2}$. Phys. Rev. Lett. 113(7), 076802 (2014).

10. S. Mouri, Y. Miyauchi, and K. Matsuda: Tunable photoluminescence of monolayer $\mathrm{MoS}_{2}$ via chemical doping. Nano Lett. 13(12), 5944 (2013).

11. J. Kim, S.S. Baik, S.H. Ryu, Y. Sohn, S. Park, B-G. Park, J. Denlinger, Y. Yi, H.J. Choi, and K.S. Kim: Observation of tunable band gap and anisotropic Dirac semimetal state in black phosphorus. Science 349(6249), 723 (2015).
12. K.I. Bolotin, K. Sikes, Z. Jiang, M. Klima, G. Fudenberg, J. Hone, P. Kim, and H. Stormer: Ultrahigh electron mobility in suspended graphene. Solid State Commun. 146(9), 351 (2008).

13. S. Larentis, B. Fallahazad, and E. Tutuc: Field-effect transistors and intrinsic mobility in ultra-thin $\mathrm{MoSe}_{2}$ layers. Appl. Phys. Lett. 101(22), 223104 (2012).

14. H. Liu, A.T. Neal, Z. Zhu, D. Tomanek, and P.D. Ye: Phosphorene: A new 2D material with high carrier mobility. arXiv preprint arXiv: 1401.4133 (2014).

15. M.M. Perera, M-W. Lin, H-J. Chuang, B.P. Chamlagain, C. Wang, X. Tan, M.M-C. Cheng, D. Tománek, and Z. Zhou: Improved carrier mobility in few-layer $\mathrm{MoS}_{2}$ field-effect transistors with ionic-liquid gating. ACS Nano 7(5), 4449 (2013).

16. W. Wu, D. De, S-C. Chang, Y. Wang, H. Peng, J. Bao, and S-S. Pei: High mobility and high on/off ratio field-effect transistors based on chemical vapor deposited single-crystal $\mathrm{MoS}_{2}$ grains. Appl. Phys. Lett. 102(14), 142106 (2013).

17. B.N. Szafranek, D. Schall, M. Otto, D. Neumaier, and H. Kurz: High on/off ratios in bilayer graphene field effect transistors realized by surface dopants. Nano Lett. 11(7), 2640 (2011).

18. H. Liu, A.T. Neal, Z. Zhu, Z. Luo, X. Xu, D. Tománek, and P.D. Ye: Phosphorene: An unexplored 2D semiconductor with a high hole mobility. ACS Nano 8(4), 4033 (2014).

19. A. Urich, K. Unterrainer, and T. Mueller: Intrinsic response time of graphene photodetectors. Nano Lett. 11(7), 2804 (2011).

20. M. Buscema, D.J. Groenendijk, S.I. Blanter, G.A. Steele, H.S. van der Zant, and A. Castellanos-Gomez: Fast and broadband photoresponse of few-layer black phosphorus field-effect transistors. Nano Lett. 14(6), 3347 (2014).

21. V. Tran, R. Soklaski, Y. Liang, and L. Yang: Layer-controlled band gap and anisotropic excitons in few-layer black phosphorus. Phys. Rev. B: Condens. Matter Mater. Phys. 89(23), 235319 (2014).

22. L. Li, J. Kim, C. Jin, G. Ye, D.Y. Qiu, F.H. da Jornada, Z. Shi, L. Chen, Z. Zhang, and F. Yang: Direct observation of layerdependent electronic structure in phosphorene. arXiv preprint arXiv:1601.03103 (2016).

23. R. Xu, J. Yang, Y. Zhu, H. Yan, J. Pei, Y.W. Myint, S. Zhang, and Y. Lu: Layer-dependent surface potential of phosphorene and anisotropic/layer-dependent charge transfer in phosphorene-gold hybrid systems. Nanoscale 8(1), 129 (2016).

24. S. Das, W. Zhang, M. Demarteau, A. Hoffmann, M. Dubey, and A. Roelofs: Tunable transport gap in phosphorene. Nano Lett. 14(10), 5733 (2014).

25. X. Peng, Q. Wei, and A. Copple: Strain-engineered direct-indirect band gap transition and its mechanism in two-dimensional phosphorene. Phys. Rev. B: Condens. Matter Mater. Phys. 90(8), 085402 (2014)

26. B. Sa, Y-L. Li, J. Qi, R. Ahuja, and Z. Sun: Strain engineering for phosphorene: The potential application as a photocatalyst. J. Phys. Chem. C 118(46), 26560 (2014)

27. A.H. Woomer, T.W. Farnsworth, J. Hu, R.A. Wells, C.L. Donley, and S.C. Warren: Phosphorene: Synthesis, scale-up, and quantitative optical spectroscopy. ACS Nano 9(9), 8869 (2015).

28. A. Ziletti, A. Carvalho, P. Trevisanutto, D. Campbell, D. Coker, and A.C. Neto: Phosphorene oxides: Band gap engineering of phosphorene by oxidation. Phys. Rev. B: Condens. Matter Mater. Phys. 91(8), 085407 (2015).

29. J. Lu, J. Wu, A. Carvalho, A. Ziletti, H. Liu, J. Tan, Y. Chen, A. Castro Neto, B. Özyilmaz, and C.H. Sow: Bandgap engineering of phosphorene by laser oxidation toward functional 2D materials. ACS Nano 9(10), 10411 (2015).

30. J. Dai and X.C. Zeng: Bilayer phosphorene: Effect of stacking order on bandgap and its potential applications in thin-film solar cells. J. Phys. Chem. Lett. 5(7), 1289 (2014). 
31. J. Padilha, A. Fazzio, and A.J. da Silva: van der Waals heterostructure of phosphorene and graphene: Tuning the Schottky barrier and doping by electrostatic gating. Phys. Rev. Lett. 114(6), 066803 (2015).

32. T. Hu and J. Hong: Anisotropic effective mass, optical property, and enhanced band gap in $\mathrm{BN} /$ phosphorene/BN heterostructures. ACS Appl. Mater. Interfaces 7(42), 23489 (2015).

33. S.P. Koenig, R.A. Doganov, L. Seixas, A. Carvalho, J.Y. Tan, K. Watanabe, T. Taniguchi, N. Yakovlev, A.H. Castro Neto, and B. Özyilmaz: Electron doping of ultrathin black phosphorus with $\mathrm{Cu}$ adatoms. Nano Lett. 16(4), 2145 (2016).

34. V.V. Kulish, O.I. Malyi, C. Persson, and P. Wu: Adsorption of metal adatoms on single-layer phosphorene. Phys. Chem. Chem. Phys. 17(2), 992 (2015).

35. T. Takagahara and K. Takeda: Theory of the quantum confinement effect on excitons in quantum dots of indirect-gap materials. Phys. Rev. B: Condens. Matter Mater. Phys. 46(23), 15578 (1992).

36. G. Qin, Q-B. Yan, Z. Qin, S-Y. Yue, M. Hu, and G. Su: Anisotropic intrinsic lattice thermal conductivity of phosphorene from first principles. Phys. Chem. Chem. Phys. 17(7), 4854 (2015).

37. Q. Wei and X. Peng: Superior mechanical flexibility of phosphorene and few-layer black phosphorus. Appl. Phys. Lett. 104(25), 251915 (2014).

38. L. Wang, A. Kutana, X. Zou, and B.I. Yakobson: Electro-mechanical anisotropy of phosphorene. Nanoscale 7(21), 9746 (2015).

39. J. Qiao, X. Kong, Z-X. Hu, F. Yang, and W. Ji: High-mobility transport anisotropy and linear dichroism in few-layer black phosphorus. Nat. Commun. 5, 4475 (2014).

40. S. Zhang, J. Yang, R. Xu, F. Wang, W. Li, M. Ghufran, Y-W. Zhang, Z. Yu, G. Zhang, and Q. Qin: Extraordinary photoluminescence and strong temperature/angle-dependent Raman responses in few-layer phosphorene. ACS Nano 8(9), 9590 (2014).

41. X. Wang, A.M. Jones, K.L. Seyler, V. Tran, Y. Jia, H. Zhao, H. Wang, L. Yang, X. Xu, and F. Xia: Highly anisotropic and robust excitons in monolayer black phosphorus. Nat. Nanotechnol. 10(6), 517 (2015).

42. R. Xu, S. Zhang, F. Wang, J. Yang, Z. Wang, J. Pei, Y.W. Myint, B. Xing, Z. Yu, and L. Fu: Extraordinarily bound quasi-onedimensional trions in two-dimensional phosphorene atomic semiconductors. ACS Nano 10(2), 2046 (2016).

43. V. Tran, R. Soklaski, Y. Liang, and L. Yang: Layer-controlled band gap and anisotropic excitons in phosphorene transport. Phys. Rev. B 89(23), 235319 (2014).

44. A. Ziletti, A. Carvalho, D.K. Campbell, D.F. Coker, and A.C. Neto: Oxygen defects in phosphorene. Phys. Rev. Lett. 114(4), 046801 (2015).

45. X. Luo, Y. Rahbarihagh, J.C. Hwang, H. Liu, Y. Du, and D.Y. Peide: Temporal and thermal stability of $\mathrm{Al}_{2} \mathrm{O}_{3}$-passivated phosphorene mosfets. IEEE Electron Device Lett. 35(12), 1314 (2014).
46. H. Zhu, S. McDonnell, X. Qin, A. Azcatl, L. Cheng, R. Addou, J. Kim, P.D. Ye, and R.M. Wallace: $\mathrm{Al}_{2} \mathrm{O}_{3}$ on black phosphorus by atomic layer deposition: An in situ interface study. ACS Appl. Mater. Interfaces 7(23), 13038 (2015).

47. J. Pei, X. Gai, J. Yang, X. Wang, Z. Yu, D-Y. Choi, B. Luther-Davies, and Y. Lu: Producing air-stable monolayers of phosphorene and their defect engineering. Nat. Commun. 7, 10450 (2016).

48. A. Avsar, I.J. Vera-Marun, J.Y. Tan, K. Watanabe, T. Taniguchi, A.H. Castro Neto, and B. Ozyilmaz: Air-stable transport in graphenecontacted, fully encapsulated ultrathin black phosphorus-based field-effect transistors. ACS Nano 9(4), 4138 (2015).

49. W. Bao, X. Cai, D. Kim, K. Sridhara, and M.S. Fuhrer: High mobility ambipolar $\mathrm{MoS}_{2}$ field-effect transistors: Substrate and dielectric effects. Appl. Phys. Lett. 102(4), 042104 (2013).

50. V. Tayari, N. Hemsworth, I. Fakih, A. Favron, E. Gaufrès, G. Gervais, R. Martel, and T. Szkopek: Two-dimensional magnetotransport in a black phosphorus naked quantum well. Nat. Commun. 6, 7702 (2015)

51. W. Zhu, M.N. Yogeesh, S. Yang, S.H. Aldave, J-S. Kim, S. Sonde, L. Tao, N. Lu, and D. Akinwande: Flexible black phosphorus ambipolar transistors, circuits and AM demodulator. Nano Lett. 15(3), 1883 (2015).

52. S. Appalakondaiah, G. Vaitheeswaran, S. Lebegue, N.E. Christensen, and A. Svane: Effect of van der Waals interactions on the structural and elastic properties of black phosphorus. Phys. Rev. B: Condens. Matter Mater. Phys. 86(3), 035105 (2012).

53. F. Wu, F. Qu, and A. MacDonald: Exciton band structure of monolayer $\mathrm{MoS}_{2}$. Phys. Rev. B: Condens. Matter Mater. Phys. 91(7), 075310 (2015)

54. J. Yang, R. Xu, J. Pei, Y.W. Myint, F. Wang, Z. Wang, S. Zhang, Z. Yu, and Y. Lu: Optical tuning of exciton and trion emissions in monolayer phosphorene. Light: Sci. Appl. 4(7), e312 (2015).

55. A. Rodin, A. Carvalho, and A.C. Neto: Excitons in anisotropic two-dimensional semiconducting crystals. Phys. Rev. B: Condens. Matter Mater. Phys. 90(7), 075429 (2014).

56. L. Li, Y. Yu, G.J. Ye, Q. Ge, X. Ou, H. Wu, D. Feng, X.H. Chen, and Y. Zhang: Black phosphorus field-effect transistors. Nat. Nanotechnol. 9(5), 372 (2014).

57. R.W. Keyes: The electrical properties of black phosphorus. Phys. Rev. 92(3), 580 (1953).

58. P. Li and I. Appelbaum: Electrons and holes in phosphorene. Phys. Rev. B: Condens. Matter Mater. Phys. 90(11), 115439 (2014).

59. N. Mao, J. Tang, L. Xie, J. Wu, B. Han, J. Lin, S. Deng, W. Ji, H. Xu, and K. Liu: Optical anisotropy of black phosphorus in the visible regime. J. Am. Chem. Soc. 138(1), 300 (2015).

60. M. Huang, M. Wang, C. Chen, Z. Ma, X. Li, J. Han, and Y. Wu: Broadband black-phosphorus photodetectors with high responsivity. Adv. Mater. 28(18), 3481 (2016).

61. J.D. Wood, S.A. Wells, D. Jariwala, K-S. Chen, E. Cho, V.K. Sangwan, X. Liu, L.J. Lauhon, T.J. Marks, and M.C. Hersam: Effective passivation of exfoliated black phosphorus transistors against ambient degradation. Nano Lett. 14(12), 6964 (2014).

\section{Supplementary Material}

To view supplementary material for this article, please visit https://doi.org/10.1557/jmr.2017.71. 